\title{
Maternal Ethanol Exposure Acutely Elevates Src Family Kinase Activity in the Fetal Cortex
}

\author{
Dandan Wang ${ }^{1,2} \cdot$ Brian W. Howell ${ }^{1} \cdot$ Eric C. Olson ${ }^{1,2}$ (i) \\ Received: 9 April 2021 / Accepted: 20 June 2021 / Published online: 16 July 2021 \\ (C) The Author(s) 2021
}

\begin{abstract}
Fetal alcohol syndrome (FAS) is characterized by disrupted fetal brain development and postnatal cognitive impairment. The targets of alcohol are diverse, and it is not clear whether there are common underlying molecular mechanisms producing these disruptions. Prior work established that acute ethanol exposure causes a transient increase in tyrosine phosphorylation of multiple proteins in cultured embryonic cortical cells. In this study, we show that a similar tyrosine phosphorylation transient occurs in the fetal brain after maternal dosing with ethanol. Using phospho-specific antibodies and immunohistochemistry, we mapped regions of highest tyrosine phosphorylation in the fetal cerebral cortex and found that areas of dendritic and axonal growth showed elevated tyrosine phosphorylation $10 \mathrm{~min}$ after maternal ethanol exposure. These were also areas of Src expression and Src family kinase (SFK) activation loop phosphorylation (pY416) expression. Importantly, maternal pretreatment with the SFK inhibitor dasatinib completely prevents both the pY416 increase and the tyrosine phosphorylation response. The phosphorylation response was observed in the perisomatic region and neurites of immature migrating and differentiating primary neurons. Importantly, the initial phosphotyrosine transient ( $\sim 30 \mathrm{~min})$ targets both $\mathrm{Src}$ and Dab1, two critical elements in Reelin signaling, a pathway required for normal cortical development. This initial phosphorylation response is followed by sustained reduction in Ser3 phosphorylation of n-cofilin, a critical actin severing protein and an identified downstream effector of Reelin signaling. This biochemical disruption is associated with sustained reduction of F-actin content and disrupted Golgi apparatus morphology in developing cortical neurons. The finding outlines a model in which the initial activation of SFKs by ethanol has the potential to disrupt multiple developmentally important signaling systems for several hours after maternal exposure.
\end{abstract}

Keywords Fetal alcohol syndrome disorder $\cdot$ Cortical development $\cdot$ Src kinases $\cdot$ Dab1 $\cdot$ Dendritogenesis $\cdot$ Cofilin

\section{Introduction}

The CDC estimates that $0.2-1.5$ per 1000 live births are children with FASD, a syndrome characterized by disrupted fetal brain development and postnatal intellectual disability (ID) [1, 2]. Disrupted connectivity including altered dendritic structure, axonal pathfinding, and white matter tracts are common findings in FAS and are thought to be major contributors to ID [3-6]. However, the cellular and biological targets of alcohol are diverse, and it is not clear whether there are common underlying molecular mechanisms producing these

Eric C. Olson

olsone@upstate.edu

1 Department of Neuroscience and Physiology, SUNY Upstate Medical University, 505 Irving Ave, Syracuse, NY 13210, USA

2 Developmental Exposure to Alcohol Research Center (DEARC), Binghamton University, Binghamton, NY 13902, USA disruptions. Identification of common molecular mechanism(s) would enable a deeper understanding of this disorder, inform studies of genetic susceptibilities, and possibly identify molecular targets for neuroprotective strategies.

This study extends our recent finding that ethanol exposure leads to SFK activation, inappropriate tyrosine phosphorylation in cultured primary neurons [7]. One consequence of the disruption is that the Reelin signaling pathway can no longer be activated in the presence of ethanol [7]. The Reelin-Dab1 signaling is absolutely required for proper cortical development [8], and genetic disruptions of this pathway are associated with disorganized cortical architecture and severe intellectual disability in humans [9]. Reelin is a secreted ligand $[10,11]$ that orchestrates correct neuronal positioning and dendritic development in many regions of the central nervous system including the cortex, hippocampus, and cerebellum. Complete absence of Reelin [10], the receptors [12], the essential adaptor protein Dab1 [13], or the Src family kinases (SFKs) Src and Fyn [14] leads to similar histological 
disruptions in the central nervous system (CNS). In humans, a lack of Reelin causes severe ID, ataxia, and mild epilepsy [9].

In prior studies, we observed similarities between ethanolexposed and Reelin signaling-deficient embryonic neurons including disrupted cellular orientation, primary dendrite numbers, and Golgi compaction $[15,16]$. Because our explant multiphoton imaging studies revealed a rapid alteration in dendritic filopodial behavior in response to acute ethanol exposure [7], we focused on biochemical events that occur within the first few minutes after ethanol exposure. This approach led to the discovery of transient increase in general tyrosine phosphorylation levels $10 \mathrm{~min}$ following ethanol exposure. This increase is blocked by the Src family kinase (SFK) inhibitors PP2 and SKI-1, but not an inhibitor of Abl tyrosine kinase [7], thereby pinpointing SFKs in the response. The transient increase is followed by a sustained period during which the pathway itself can no longer be activated by in vitro application of the ligand, Reelin. Thus, ethanol appears to disrupt SFK and Reelin signaling in vitro.

In this study, we show that a similar disruption of SFKdependent phosphorylation occurs in the fetal brain after maternal ethanol exposure. The robust nature of the ethanolinduced tyrosine phosphorylation response allowed us to use immunohistochemistry (IHC) to map changes of phosphorylated tyrosine content in fetal brain sections. The areas of maximal change include the marginal zone (MZ) where Reelin-dependent dendritogenesis occurs and the intermediate zone (IZ) that contains the forming white matter tracts. These areas also correlate with areas of high Src protein expression and Src activation (pY416 activation loop immunosignal). We find that maternal ethanol exposure first causes aberrant $\mathrm{Src}$ activation and Dab1 phosphorylation followed by sustained ncofilin dephosphorylation and Golgi apparatus disruption. This suggests that maternal ethanol exposure may significantly impair SFK and Reelin signaling in the fetal brain.

\section{Material and Methods}

\section{Animals}

All animal procedures were performed in accordance with the Institutional Animal Care and Use Committee of SUNY Upstate Medical University. C57BL/6J mice (Jackson Laboratory) were used in this study. The day of plug discovery is considered embryonic day 0 (E0). Pregnant C57BL/6J were housed in a temperature-controlled room with a 12:12-h light-dark cycle. Intraperitoneal injections of pregnant dams were performed on E15 with either control phosphatebuffered saline (PBS) or PBS/ethanol to achieve $4 \mathrm{~g}$ ethanol/ $\mathrm{kg}$ bodyweight, equivalent to $0.5 \% \mathrm{v} / \mathrm{v}$. In some experiments, the Src family kinase inhibitor dasatinib $(20 \mathrm{mg} / \mathrm{kg}$ bodyweight) was injected $30 \mathrm{~min}$ prior to ethanol or control injections. Animals were sacrificed 10, 30, 120, and $240 \mathrm{~min}$ after injection. Individual embryonic brains were rapidly isolated. Half of the brain was drop fixed in a $4 \%$ paraformaldehyde (PFA) PBS solution and then cryoprotected in 30\% sucrose before cryostat sectioning for later histological analysis. The dorsal cortex of the other half brain was dissected for subsequent western blot analysis.

\section{Blood Ethanol Concentration and Fetal Amniotic Ethanol Concentration}

Tail blood was collected in heparin coated tubes 10 min prior to the ethanol injections to establish a baseline, and then blood was taken at $10 \mathrm{~min}, 30 \mathrm{~min}, 1 \mathrm{~h}, 2 \mathrm{~h}$, and $4 \mathrm{~h}$ after injection. Amniotic fluid was collected immediately after the injected dam had been sacrificed and the uterus exposed. A microsyringe was used to puncture the uterine wall and amniotic sac. A small volume of amniotic fluid was then withdrawn from the amniotic sac for subsequent analysis. The maternal blood and fetal amniotic fluid samples were then centrifuged at $2000 \mathrm{rpm}$ for $15 \mathrm{~min}$ and stored at $-80^{\circ} \mathrm{C}$ prior to analysis with an Analox Alcohol Analyzer (Analox Instruments, Lunenburg, MA).

\section{Immunohistochemistry}

The $30 \mu \mathrm{m}$ coronal cryosections were applied to glass slides and allowed to dry at room temperature overnight. Tissue sections were washed in phosphate-buffered saline (PBS) for $15 \mathrm{~min}$ and then blocked in 5\% donkey serum, $1 \%$ BSA, and $0.2 \%$ Triton $\mathrm{X}-100$ for $1 \mathrm{~h}$ at room temperature and incubated with primary antibody at $4{ }^{\circ} \mathrm{C}$ overnight. Mouse anti-pY99 (1:1000, Santa Cruz), rabbit antiMAP2 (1:200, Protein Tech Co.), rabbit anti-pY416 (1:200, Cell Signaling), rabbit anti-pan Src antibody (1:200, Santa Cruz), rabbit anti-Dcx (1:200, [17], rabbit anti-Tbr1 (1:200, Abcam), rabbit anti-GAD67 (1:200, Millipore), and mouse anti-GM130 (1:200, BD) were used as primary antibodies. Donkey anti-species IgG conjugated with Alexa 488, Alexa 555 or Alexa 647, and Alexa 555-conjugated phalloidin were used as secondary antibodies. Hoechst 33342 ( $2 \mu \mathrm{g} / \mathrm{ml}$, Molecular Probes) was used to visualize individual cell nuclei. Images were collected with a Zeiss LSM780 laser scanning confocal microscope (Advanced Fluorescence Imaging Core, SUNY Upstate Medical University). To compare the tyrosine phosphorylation response after different treatment, the average pY99 signal intensity was collected and normalized to the MAP2 immunosignal intensity and then compared among different treated group. 


\section{Cortical Cultures and Treatments}

Cortical tissue (E15) was dissociated using $0.25 \%$ trypsin at $37{ }^{\circ} \mathrm{C}$ for $20 \mathrm{~min}$. A 10:10 buffer (10\% trypsin inhibitor and $10 \%$ Bovine Serum Albumin, both from Sigma) was used to neutralize trypsin. Neurons were resuspended in Neurobasal medium supplemented with 2\% B27, $1 \%$ GlutaMAX, and $1 \%$ penicillin/streptomycin (all from Invitrogen) and plated onto PDL-coated glass coverslips in 24-well plates. After 3 day in vitro (DIV), cultures were exposed to ethanol $(400 \mathrm{mg} /$ $\mathrm{dL}, 0.5 \% \mathrm{v} / \mathrm{v}$ ) or PBS and then fixed for subsequent immunocytochemistry. For western blot analysis, cortical cultures were plated on PDL-coated 12-well tissue culture plates at a final concentration of $2 \times 10^{6}$ cells $/ \mathrm{ml}$ and then cultured for another $72 \mathrm{~h}$. Lysates were collected after treatment with ethanol $(400 \mathrm{mg} / \mathrm{dL}, 0.5 \% \mathrm{v} / \mathrm{v})$ or $\mathrm{H}_{2} \mathrm{O}$.

\section{Western Blot Analysis}

Both adult and E15 embryonic dorsal cortex and primary cortical neuron lysates were collected in radio immunoprecipitation assay (RIPA) buffer (50 mM Tris-HCl; $1 \% \mathrm{NP}-40 ; 0.25 \%$ Na-deoxycholate; $150 \mathrm{mM} \mathrm{NaCl} ; 1 \mathrm{mM}$ EDTA, pH 7.4) with protease inhibitors (Protease Inhibitor Cocktail, Sigma) and phosphatase inhibitors $\left(1 \mathrm{mM} \mathrm{Na}_{3} \mathrm{VO}_{4} ; 1 \mathrm{mM} \mathrm{NaF}\right)$ on DIV 3. All samples were loaded onto $10 \%$ SDS-PAGE gels and transferred to $0.45 \mu \mathrm{m}$ PVDF Immobilon®-FL membranes (EMD Millipore). After incubation with Odyssey blocking buffer (LI-COR Biosciences) for $1 \mathrm{~h}$ at room temperature, the membranes were incubated with the following primary antibodies overnight at $4{ }^{\circ} \mathrm{C}$. The primary antibodies were anti-pY99 (1:1000, Santa Cruz), anti-pY416 (1:1000, Invitrogen), anti-pan Src antibody (1:1000, Millipore), antiDab1 (1:1000, Sigma), anti-p-Cofilin (Ser3, 1:1000, Invitrogen), anti-Cofilin (1:1000, SantaCruz) and antiGAPDH (1:2000, UBPBio) were used as loading controls. Appropriate secondary antibodies IRDye ${ }^{\circledR} 800 \mathrm{CW}$ and IRDye ${ }^{\circledR}$ 680RD (LI-COR Biosciences) were used, and membranes were scanned using the Odyssey ${ }^{\circledR}$ CLx system (LICOR Biosciences).

\section{Ex Utero Electroporation and Explant Cultures}

Explant preparation and ex utero electroporation were performed on E13 embryos using $0.8 \mathrm{mg} / \mathrm{ml}$ of pCAG-GFP construct [15]. Hemispheres were dissected and cultured medial side down on $3 \mu \mathrm{m}$ pore size, collagen-coated, polytetrafluoroethylene filters (Transwell-COL, Corning) in DMEM-F12 media plus GlutaMAX and supplemented with $1 \% \mathrm{G} 5,2 \% \mathrm{~B} 27$, and $1 \%$ penicillin/streptomycin (all from Invitrogen). Explant cultures were maintained in a high oxygen environment $\left(95 / 5 \% \mathrm{O}_{2} / \mathrm{CO}_{2}\right)$ at $37{ }^{\circ} \mathrm{C}$ for $48 \mathrm{~h}$ before ethanol or control treatments. Explants from both control and ethanol groups were drop fixed in 4\% PFA for subsequent histological analysis.

\section{Golgi Morphology Measurement}

To investigate the effect of ethanol on Golgi complex continuity and morphology, we used the Dcx-dsRed ${ }^{14 Q 1 u / J}$ transgenic mouse that expresses dsRed fluorescent protein from a Doublecortin (Dcx) promoter element [18]. The mouse provides genetic identification of developing cortical neurons. The number of puncta and the proximal to distal Golgi length were measured within dsRed $^{+}$neurons by tracing the GM130 immunosignal through the imaged $\mathrm{z}$-series of the cell. Differentiating neurons were identified as those cells with soma located in the cortical plate and a leading process contacting the marginal zone, whereas migrating neurons were identified as those cells with migrating morphology and a leading process that did not contact the MZ.

\section{Experimental Design and Statistical Analysis}

Graphing and statistical analyses were performed in GraphPad Prism 7. A minimum of two embryos from at least three dams were analyzed for each experiment in each condition. Data are presented as the mean \pm SEM. Experiments are designed to determine differences between two groups (control vs. ethanol exposed) using the Student's t-test. For densitometery and time responses involving multiple measurements, one or two-way ANOVA with post hoc Bonferroni tests was used.

\section{Results}

\section{Ethanol Increases Tyrosine Phosphorylation After Maternal Exposure}

In our previous study, we found that phosphotyrosine levels in lysates of primary cortical cultures derived from Swiss Webster (SW) embryos was transient and largely absent after 30 min of continuous ethanol exposure, a timeline that was paralleled by the in vivo response. To further explore the in vivo response, we used C57B16/J mice, a commonly used background used for genetic manipulation and for FAS studies. To provide precise temporal control of the exposure, pregnant dams received an intraperitoneal (i.p.) injection of ethanol $(4 \mathrm{~g} / \mathrm{kg}$ predicted to achieve $\sim 400 \mathrm{mg} / \mathrm{dL})$ or PBS on E15. The ethanol dose is within the range of ethanol exposures that have been used in alcohol abuse studies [19, 20] [21] and corresponds to blood ethanol concentration (BECs) that can be found in human subjects with alcohol abuse disorder [22, 23]. The injected dams typically remained conscious during the dosing period, and maternal BEC measurements reached $250 \mathrm{mg} / \mathrm{dL}$ at $60 \mathrm{~min}$ post injection declining to $116 \mathrm{mg} / \mathrm{dL}$ by 
$4 \mathrm{~h}$ after injection. In contrast, amniotic fluid EC achieved a concentration of $430 \mathrm{mg} / \mathrm{dL}, 10 \mathrm{~min}$ after injection, the predicted value based on the injection and similar to $400 \mathrm{mg} / \mathrm{dL}$ dose used in our prior in vitro study [7]. Four hours after injection the amniotic fluid showed an EC of $120 \mathrm{mg} / \mathrm{dL}$, equivalent to maternal BEC (see Supplemental Figure). The dams were then euthanized, the embryos were removed, and the embryonic brain was split sagitally: One hemi-brain was drop fixed in $4 \%$ paraformaldehyde for subsequent IHC analyses, and the remaining hemi-brain was further dissected in ice cold saline to isolate dorsal neocortex, which was then used to generate lysates for western blot analyses.

Elevated phosphotyrosine (pY99) levels were observed in fetal cortical lysates at $10 \mathrm{~min}$ after maternal exposure and persisted for at least $30 \mathrm{~min}$ before returning to baseline by $4 \mathrm{~h}$ (Fig. $1 \mathrm{~A}$ and B). The $\sim 1.5$-fold increase in total pY99 signal was in the same direction but smaller in magnitude than the $\sim 3.5$-fold increase in pY99 signal observed with SW. However, the C57B16/J response was sustained and was detected at $30 \mathrm{~min}$, whereas the $\mathrm{SW}$ response had returned to baseline by this time. This difference in response profile appears to be intrinsic to the neuronal cells as the same approximate timeline differences were observed in dissociated cell cultures prepared from the two strains [7] (Fig. $5 \mathrm{~A}$ and B). The acute ethanol response may be a strain-specific difference like those observed with rodent ethanol consumption [24] and fetal response to exposure [25]. Interestingly, a tyrosine phosphorylation response was not observed in lysates prepared from the maternal cortex (Fig. 1C), suggesting that embryonic neural tissue is particularly responsive to exposure. Cell death has been associated with ethanol exposure [26]. Although we found no reduction of cell viability with $400 \mathrm{mg} / \mathrm{dL}$ dose in

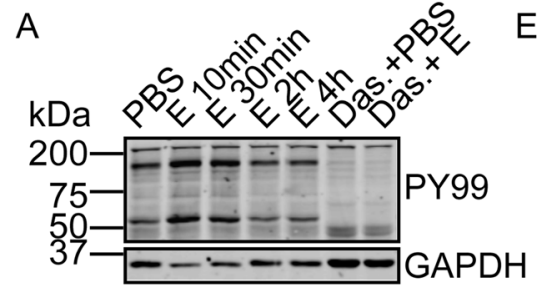

B

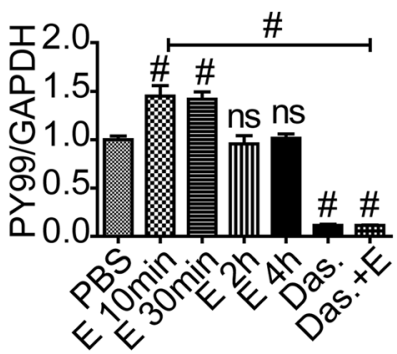

$\mathrm{F}$
C

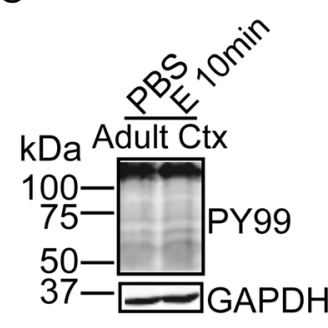

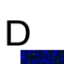

PBS 4h

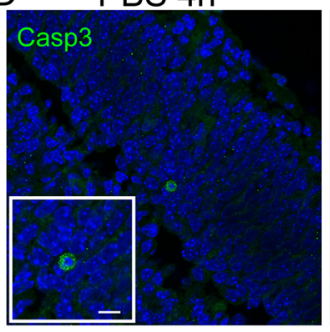

Fig. 1 Maternal ethanol exposure induces transient tyrosine phosphorylation in vivo. A Western blot detection of tyrosine phosphorylation level in E15 embryonic cortical lysate after PBS or 10, 30, 120, and $240 \mathrm{~min}$ of maternal ethanol exposure (i.p., $4 \mathrm{~g} / \mathrm{kg}$ ). B Quantification of anti-phosphotyrosine (pY99) signal shows a sustained increase in fetal cortical phosphotyrosine levels for $30 \mathrm{~min}$ after maternal exposure that is completely blocked by maternal pretreatment with the SFK inhibitor dasatinib $(20 \mathrm{mg} / \mathrm{kg})$. Densitometric values are expressed as total pY99/GAPDH and then normalized to the control (PBS-injected) group for each timepoint. C Western blot detection of tyrosine phosphorylation level in maternal cortical lysate after $10 \mathrm{~min}$ of PBS or ethanol
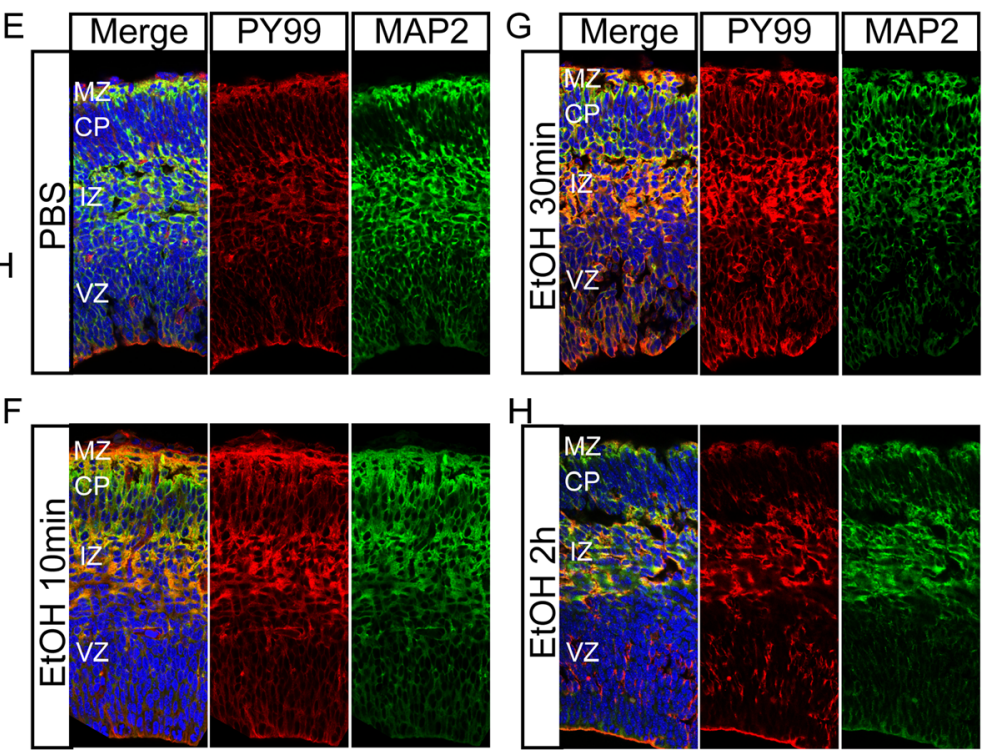
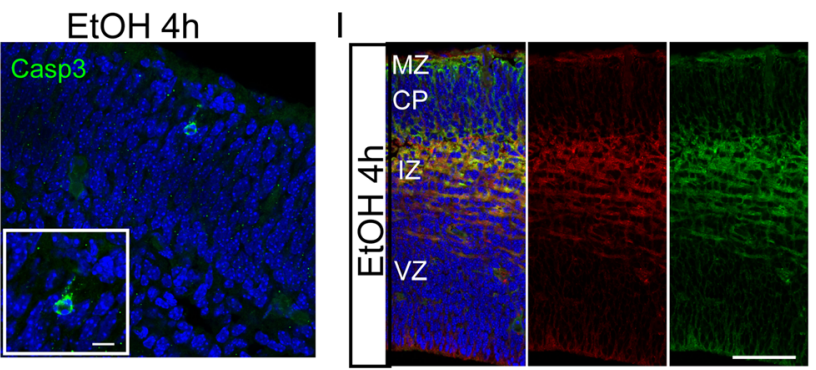

exposure. D Cleaved caspase 3 staining of E15 embryonic brain after $4 \mathrm{~h}$ of PBS or ethanol injection revealed few immunopositive cells in either condition. Scale bar, $10 \mu \mathrm{m}, \mathbf{E}-\mathbf{I}$ pY99 and anti-MAP2 immunostaining in E15 cerebral cortex after E PBS or F $10 \mathrm{~min}, \mathbf{G} 30 \mathrm{~min}, \mathbf{H} 120 \mathrm{~min}$, and I 240 min of ethanol exposure. Prominent increases in pY99 signal are observed at 10 and $30 \mathrm{~min}$ in marginal zone (MZ), the area of apical dendrite projection, and the intermediate zone (IZ), the area of axonal projection. Cortical plate (CP), ventricular zone (VZ). Scale bar, 100 $\mu \mathrm{m}$. Statistical determination by one-way ANOVA followed by Bonferroni's post hoc tests between groups: ${ }^{\#} p<0.001$ 
culture [7], sections of fetal brains were examined for evidence of cell death using cleaved caspase 3 immunohistochemistry. Caspase $3^{+}$cells were infrequently detected in either condition, and no difference in positive cells was identified between conditions $4 \mathrm{~h}$ after exposure (Fig. 1D). This finding shows that C57B16/J embryos, like SW embryos, demonstrate a rapid tyrosine phosphorylation response to acute maternal ethanol exposure.

To determine whether the in vivo fetal cortical response depended on SFK activation, we pretreated pregnant dams with dasatinib (BMS-354825). Dasatinib is an FDAapproved inhibitor of SFKs, with additional inhibition of BCR-ABL [27] [28], and is used for treatment of chronic myelogenous leukemia [29]. Dasatinib (final concentration of $20 \mathrm{mg} / \mathrm{kg}$ ) was i.p. injected into the dam $30 \mathrm{~min}$ before ethanol or PBS treatment (also i.p. injected). Dasatinib pretreatment completely blocked ethanol-induced increases in tyrosine phosphorylation (Fig. 1 A and B). In addition, dasatinib pretreatment followed by PBS injection lowered pY99 content below baseline (untreated) suggesting that the pY99 signal in the neocortex at E15 may largely be dependent on ongoing SFKs activity.

\section{Elevated Phosphotyrosine Levels Are Found in Areas of Active Neurite Growth}

We used anti-phosphotyrosine (pY99) as well as antimicrotubule-associated protein 2 (MAP2) IHC to determine the spatial and temporal patterns of increased tyrosine phosphorylation in the developing cortex. Drop-fixed hemi-brains were cryosectioned and immunostained for subsequent confocal microscopy. Ethanol caused prominent pY99 signal increases in the apical area of the ventricular zone (VZ), where neural precursors are localized, the intermediate zone (IZ) that contains migrating neurons and developing axonal tracts, the subplate (SP) and marginal zone (MZ) that contain transient populations of neurons critical for early cortical patterning (Fig. 1E-I). Importantly, for this study, the MZ also is the initial projection field for the apical dendrite of most developing excitatory neurons, the cell class responsive to Reelin signaling. Consistent with the western blot analyses, the pY99 IHC signal was elevated for $30 \mathrm{~min}$ after maternal exposure. To quantify the regional $10 \mathrm{~min}$ response, mean fluorescence intensity from regions of interest (ROIs) located in the MZ, CP (cortical plate), IZ, and ventricular zone and the subventricular zone (VZ/SVZ) were compared with corresponding regions in fetal cortices from PBS exposed dams. As shown in Fig. 2 A and B, ethanol increased pY99 reactivity across the cerebral wall compared to PBS-exposed controls, with a 1.5-fold and higher elevations in the VZ, IZ, and MZ (Fig. 2D). In contrast, MAP2 content was not altered by 10 min of ethanol exposure (not shown). The areas of greatest increase corresponded to areas of highest control pY99 levels, and the response in all areas was blocked by pretreatment of the dam with dasatinib (Fig. 2C and D).

\section{Areas of Increased Tyrosine Phosphorylation Are Also Areas of Src Kinase Expression}

In our previous study, 10 min of ethanol exposure caused an increase in activation loop phosphorylation of Src but not the related kinase Fyn, indicating that Src activity is involved in the response [7]. To determine whether the regions of maximal phosphotyrosine response correspond to regions of highest Src expression and Src activation, we performed IHC for total Src and Src pY416 activation loop epitope expression. Total Src and activated Src immunoreactivity was found prominently in the VZ, IZ, and $\mathrm{MZ}$ with highest expression in the IZ and low expression in the CP (Fig. 3 A and B). The pY416 signal strongly reduced by pretreatment with dasatinib assayed both by IHC (Fig. 3A) and by western blot (Fig. $3 \mathrm{D}$ and E). The spatial distribution of total Src and active Src immunosignal parallels the spatial distribution of maximal pY99 immunoreactivity (Fig. 3C), consistent with the hypothesis that Src itself is a major contributor to the acute response.

\section{Migrating Neurons Are Sensitive Targets for Ethanol Exposure}

To determine the cell classes generating the elevated phosphotyrosine response to ethanol, we used ex utero electroporation of CAG-GFP expression plasmid to label developing cortical cells. Electroporations were performed at E13, and whole hemisphere explants were prepared [30]. After 2 $\mathrm{DIV}, \mathrm{GFP}^{+}$cells are found at different stages of migration and development across the cerebral wall. PBS or ethanol (400 $\mathrm{mg} / \mathrm{dL}$ ) was added to the culture medium for $10 \mathrm{~min}$, and explants were immediately drop fixed for subsequent histological analyses. Consistent with our findings from in utero exposed embryos, ethanol-treated explants showed significantly more pY99 signal in the VZ, IZ, and MZ compared to control-treated explants (Fig. 4A-C vs. 4D-F). In addition to the increased pY99 signal in the apical junction of the VZ (not shown), cells with multipolar neuron morphology in the IZ showed enhanced pY99 signal in the periphery of the soma and neurites after ethanol exposure compared to control (Fig. 4 and F). While $\mathrm{GFP}^{+}$somata within the $\mathrm{CP}$ showed variable increases in pY99 in response to ethanol, dendrites projecting into the MZ showed a consistent pY99 response (Fig. 4 B and E).

To determine the magnitude and time course of the response of primary cultures derived from C57B16/J embryos, lysates were prepared at defined times after dosing with ethanol $(400 \mathrm{mg} / \mathrm{dL})$ or an equivalent volume of water. Consistent 
with our prior findings from primary cultures derived from SW embryos [7], a large increase in pY99 signal is observed at $10 \mathrm{~min}$; however, in contrast to SW cultures, the pY99 elevation persists for at least $30 \mathrm{~min}$ in cells derived from C57Bl6/J embryos (Fig. 5 A and B). This culture finding is consistent with our finding from in utero dosed embryos and suggests a cell intrinsic difference in the temporal response that depends on strain.

To confirm neurite expression of pY99 in immature excitatory neurons, cortical cultures were stimulated for $10 \mathrm{~min}$ with ethanol (400mg/dL) or $\mathrm{H}_{2} \mathrm{O}$ and then fixed and processed for immunocytochemistry. Cortical neurons were immunostained with pY99 and anti-Doublecortin (Dcx), a microtubule associated protein expressed by immature neurons [17] (Fig. 5 C and D), or separately Tbr1, a transcription factor that identifies developing excitatory neurons [31] (Fig. 5 E and F). Ethanol exposure caused a rapid response in pY99 signal on both $\mathrm{Dcx}^{+}$and $\mathrm{Tbrl}^{+}$neurons (arrows) with prominent expression in the perisomatic region and along the neurites. In many neurons, additional signal was observed in cytoplasm and in proximal region of neurites possibly reflecting organelle associated pY. Interestingly, not all neurons (stars) demonstrated a pY99 response, with $62 \%$ of $\operatorname{Dcx}^{+}(n=110)$ and $64 \%$ of $\mathrm{Tbr}^{+}$cells $(\mathrm{n}=80)$ exhibiting pronounced pY99 signal.

To determine whether GABAergic interneurons were similarly responsive to ethanol, cultures were prepared from lateral and medial ganglionic eminence tissue to enrich for interneuron populations. After $2 \mathrm{DIV}$, cultures were treated for
10 min with either ethanol (400 $\mathrm{mg} / \mathrm{dL})$ or $\mathrm{H}_{2} \mathrm{O}$ and then fixed and immunostained with GAD67 to identify interneurons and separately pY99 to reveal the phosphorylation response. Ethanol caused a detectable increase in pY99 content in $47 \%$ of GAD $67^{+}$interneurons (Fig. $5 \mathrm{G}$ and $\mathrm{H}$ ), a figure that is similar to the percentage of $\mathrm{Dcx}^{+}$and $\mathrm{Tbr} 1^{+}$cells that show an ethanol response. These findings confirm that developing excitatory and inhibitory neurons are sensitive to the acute effects of ethanol and indicate differential sensitivity or differences in the timing of the ethanol response between cells.

\section{Reelin Signaling Is Disrupted in Fetal Cortex After Maternal Ethanol Exposure}

Normally, Reelin binding to its receptors leads to SFK activation, Dab1 tyrosine phosphorylation, and the subsequent phosphorylation of downstream elements including Ser3 on the actin severing protein n-cofilin ([32], a). In vitro, ethanol exposure initially activates Src and downstream tyrosine phosphorylation but ultimately blocks Reelin signaling and causes n-cofilin Ser3 dephosphorylation in vitro [7]. To determine whether these Reelin signaling components are disrupted by in utero exposure, and the time course of that disruption, cortical lysates were prepared from embryos at defined times after maternal injection (i.p. $400 \mathrm{mg} / \mathrm{dl}$ ). Consistent with our in vitro findings, Src and an $80 \mathrm{kD}$ protein, Dab1, show aberrant elevated tyrosine phosphorylation for at least $30 \mathrm{~min}$ after exposure (Fig. 6A-C). This indicates that acute ethanol exposure inappropriately activates the Reelin

Fig. 2 Spatial expression pattern of tyrosine phosphorylation in embryonic cortex 10 min after maternal ethanol exposure with or without dasatinib. Coronal sections of E15 cortex from in utero exposed embryos were immunostained for phosphotyrosine (pY99) and MAP2. In comparison to A PBS exposed embryos, B ethanol exposed embryos (i.p. $4 \mathrm{~g} / \mathrm{kg}$ ) showed significant increases in pY99 content in the VZ, IZ, and MZ. C Pretreatment of the dam with $20 \mathrm{mg} / \mathrm{kg}$ dasatinib completely blocks the pY99 increase. Scale bar, $100 \mu \mathrm{m}$. D Densitometric quantification of the immunosignal. MAP2 immunosignal is used as an internal control. Statistical determination by one-way ANOVA followed by Bonferroni's post hoc tests between groups. ${ }^{*} p<$ $0.05, * * p<0.01, \stackrel{\#}{p}<0.001$
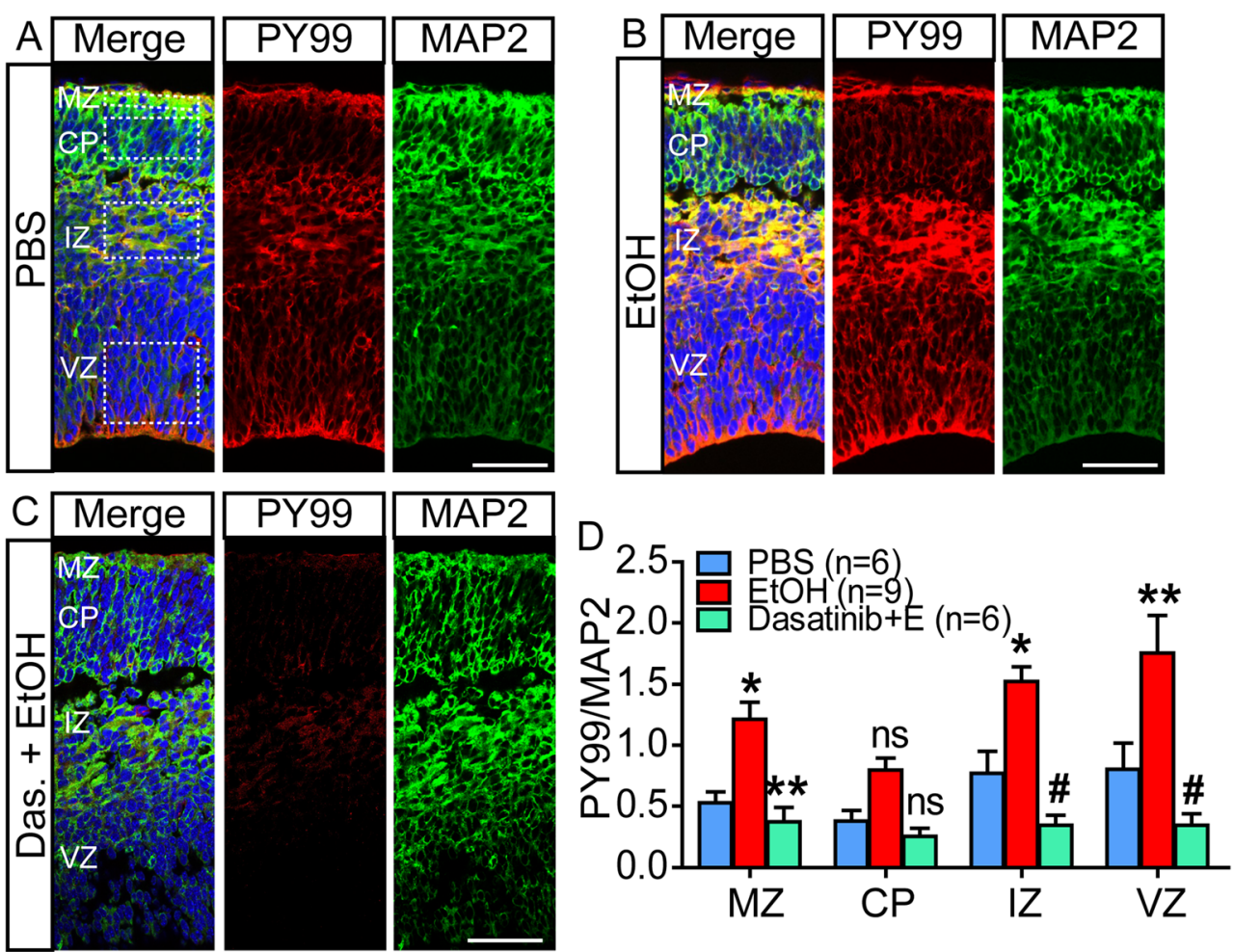
Fig. 3 Correspondence between areas of Src activation and pY99 increases after ethanol exposure. A-B Representative images of A Src activation loop (pY416) and B anti-Src immunostaining in E15 cerebral cortex 10 min after intraperitoneal injection of dams with PBS or ethanol $(4 \mathrm{~g} / \mathrm{kg})$ and with or without dasatinib $(20 \mathrm{mg} / \mathrm{kg})$ pretreatment. Scale bar, $100 \mu \mathrm{m}$. C Mean fluorescence intensity measurements show prominent Src expression and Src activation in the MZ, IZ, and VZ all areas that show significant $\mathrm{pY} 99$ response to ethanol. D Western blot of cortical lysates probed with pY416, Src, and GAPDH. A significant increase in Src activation loop signal is observed after $10 \mathrm{~min}$ ethanol exposure which is completely blocked by dasatinib $(20 \mathrm{mg} / \mathrm{kg})$ pretreatment. Samples presented in triplicate. $\mathbf{E}$ Quantification of blots in (D). Statistical determination by oneway ANOVA followed by Bonferroni's post hoc tests between groups. ${ }^{*} p<0.001$
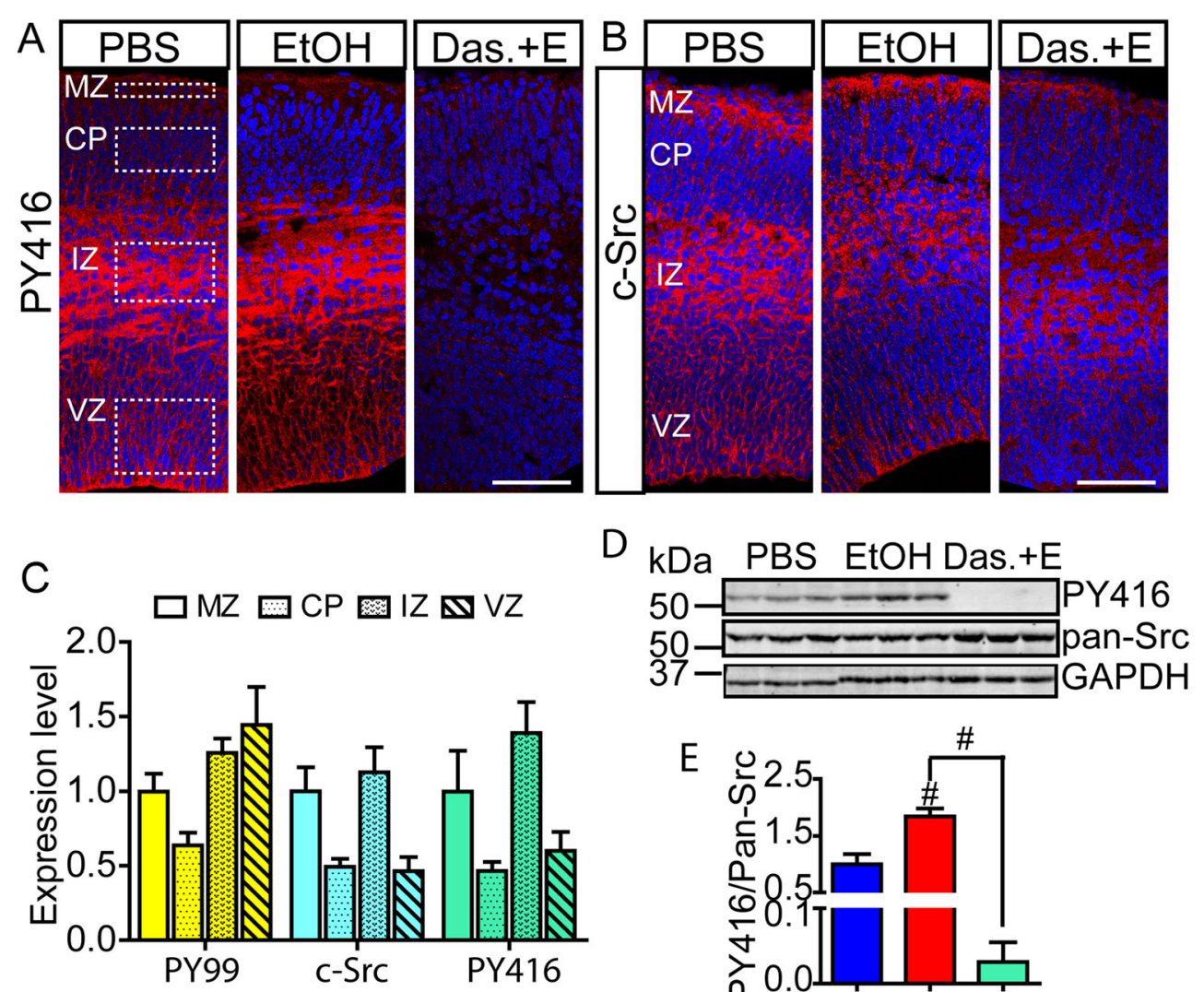

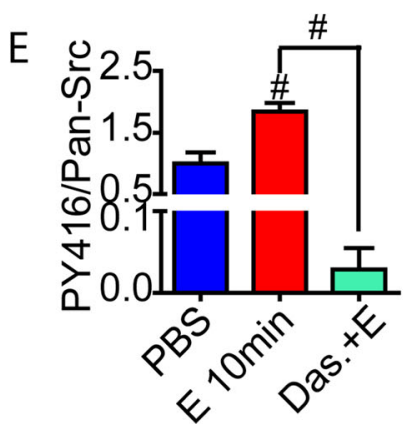

signaling pathway in vivo. As with our prior study, n-cofilin shows aberrant Ser3 dephosphorylation at $4 \mathrm{~h}$ after exposure (Fig. $6 \mathrm{~A}$ and D) suggesting that acute ethanol exposure may disrupt actin dynamics in the fetal cortex for a 4-h period after maternal exposure. Pharmacological inhibition of SFKs by dasatinib pretreatment alone also caused cofilin Ser3 dephosphorylation suggesting that the response of cofilin after ethanol exposure may possibly represent longer term SFK inhibition by ethanol as we found in vitro [7] but that is not reflected by pY416 status in vivo (Fig. $6 \mathrm{~A}$ and $\mathrm{C}$ ).

\section{Altered Cytoskeletal Protein Expression and Golgi Complex Morphology After Ethanol Exposure}

Dephosphorylation of Ser3 on n-cofilin is known to increase filamentous-actin (F-actin) severing activity [33, 34] and would be expected to alter F-actin content in the cortex. Our prior studies have shown ethanol-dependent disruption of dendrites and altered expression F-actin, MAP2, and Golgi complex morphology both in culture and in explants $[7,16]$. To determine whether similar disruptions occur in vivo after maternal exposure, sections were stained with Alexa 555phalloidin to detect F-actin and immunostained with anti-
MAP2 to detect neurites (Fig. 7 A and B). Consistent with these prior studies, both phalloidin and MAP2 signals showed a pronounced decrease in expression by $2 \mathrm{~h}$. This effect was observed across the cerebral wall including the $\mathrm{MZ}$ and IZ, but also the SVZ, an area of active neuronal proliferation (Fig. 7C-F). Interestingly, the difference in phalloidin signal was observed at $30 \mathrm{~min}$ of exposure, whereas the difference in MAP2 signal was detected at $2 \mathrm{~h}$ suggesting that microtubule cytoskeletal alterations may follow actin disruptions.

The Golgi complex performs multiple essential functions in the developing cortical neurons [35, 36] [37] and can be morphologically and functionally regulated by Src signaling [38]. We and others have shown reduced extension of Golgi apparatus into the apical dendrite after ethanol exposure [16, 39] and after Reelin signaling is disrupted [15, 40-42]. Consistent with these prior studies that employed explants and cell culture models, maternal ethanol exposure also caused sustained ( $4 \mathrm{~h}$ ) disruption of Golgi complex morphology in fetal cortical neurons with increased fragmentation and less extension into the apical dendrite (Fig. 7G-L). These findings establish a temporal sequence of ethanol-dependent disruptions and shows that these effects can impact fetal neurons for hours after initial maternal exposure. 

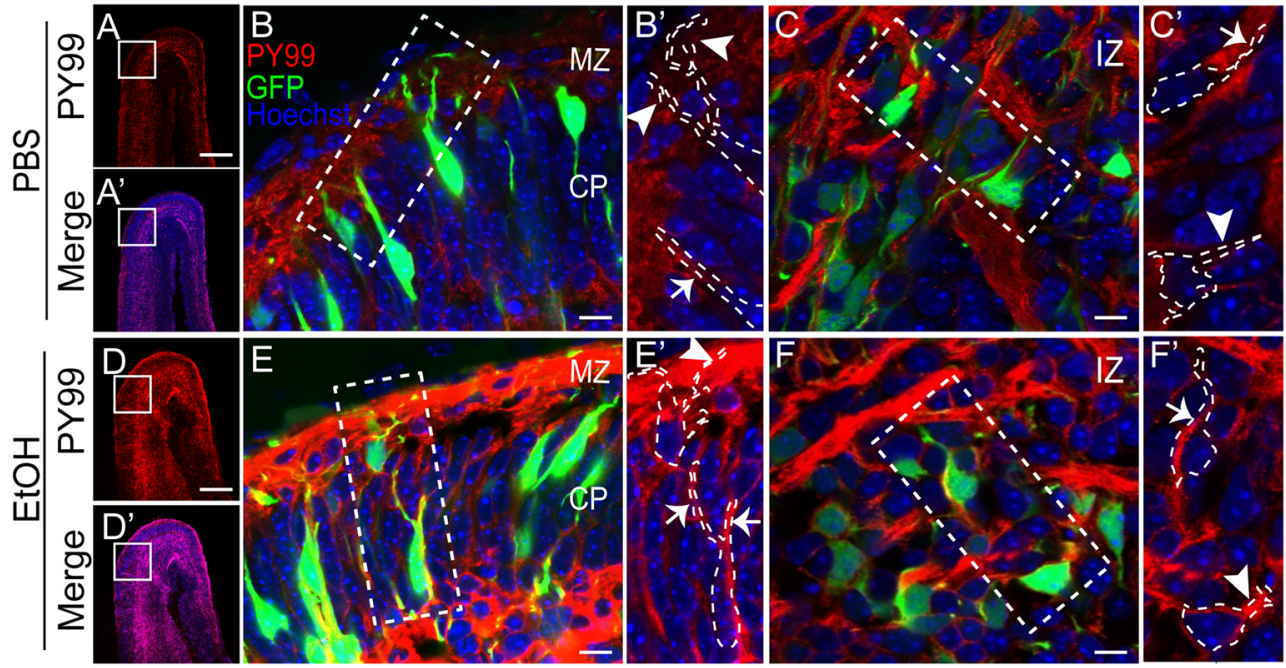

Fig. 4 Migrating and differentiating cortical excitatory neurons are sensitive to acute ethanol exposure. Prospective deep layer excitatory neurons were targeted by E13 ex utero electroporation of dorsal neocortex with a GFP expression plasmid. Whole hemisphere explants were cultured on collagen-coated filters for 2 DIV. Explants were then treated with A-C PBS or D-F $400 \mathrm{mg} / \mathrm{dL}$ ethanol for $10 \mathrm{~min}$. In comparison to A PBS-injected control, significantly higher pY99 immunosignal

is observed in $\mathbf{D}$ ethanol-exposed explants. Higher magnification views of B,C PBS and $\mathbf{E}, \mathbf{F}$ ethanol-exposed explants revealed increased pY99 immunosignal in cell bodies and neurites of migrating and differentiating neurons in the CP and multipolar neurons in the IZ. The insets show traced outlines of the $\mathrm{GFP}^{+}$cells and the corresponding pY99 signal in those regions. Sections were counterstained with Hoechst. Scale bar for (A) and (D) is $100 \mu \mathrm{m}$. Scale bar for $(\mathbf{B}),(\mathbf{C}),(\mathbf{E})$, and $(\mathbf{F})$ is $10 \mu \mathrm{m}$

\section{Discussion}

In this study, we provide evidence that maternal exposure to ethanol leads to rapid activation of Src family kinases and an elevation of tyrosine phosphorylation in multiple fetal cortical regions and cell types. Importantly, this brief phosphorylation increase is followed by an extended period of at least $2 \mathrm{~h}$ in which the Reelin signaling effector n-cofilin is dephosphorylated and F-actin content across the cerebral wall is reduced. In addition, we observed disrupted Golgi apparatus morphology that is similar to that observed with deficiency in Reelin signaling. These findings suggest that ethanol exposure may selectively impact SFK-dependent signaling pathways in the fetal cortex after maternal ethanol exposure.

Our current findings both in vitro and in vivo outline a model in which transient SFK-activation by ethanol initiates a sequence of events that potential could impact signaling for extended periods of time. During the first phase of the ethanol response, Src is activated causing the tyrosine phosphorylation of its own activation loop, and Dab1, an adaptor protein essential for Reelin signaling. Our western blot analyses reveal that activation of SFKs by ethanol causes non-specific phosphorylation of multiple other protein species besides Dab1 at $\sim 80 \mathrm{kD}$ and Src itself at $\sim 57 \mathrm{kD}$ and this response is distinct from the relatively specific activation of Src and Dab1 by Reelin $[43,44]$. Although the identity of these other proteins is currently unknown, this inappropriate tyrosine phosphorylation likely indicates the dysregulation of multiple signaling pathways besides Reelin.

SFKs are essential components of several developmentally important signaling pathways [45] [46] [47]. While mice singly deficient in the SFKs Src and Fyn do not show obvious brain malformations [14], mice singly deficient in Src exhibit small size and osteopetrosis (increased bone density) [48]. In contrast, mice doubly deficient in Src and Fyn show the reeler cortical phenotype and profound layering and dendritic disruptions during early cortical development $[14,49]$. This suggests some redundancy in SFK signaling but also highlights that inactivation of both SFKs blocks Reelin signaling during early cortical development.

The elevation of phosphotyrosine content by ethanol exposure is relatively brief ( $5-30 \mathrm{~min})$ and is followed by a tyrosine dephosphorylation phase when total phosphotyrosine levels drop to baseline. During the latter period in vitro, application of Reelin protein could not initiate Reelin signaling, and no increase in Dab1 phosphorylation could be elicited [7]. During this period in vivo, pY416 levels remained at baseline, and since this antibody recognizes both Src and Fyn activation domains, both SFKs may be inactive during the latter phase of ethanol exposure. Inactivation of both SFKs could produce the phenotypic convergence between Reelin deficiency and ethanol exposure. Consistent with a sustained inactivation of Reelin signaling, we found that Ser3 of n-cofilin did drop below baseline indicating a sustained activation of this actin severing protein that is normally inactivated by Reelin signaling $[32,50]$.

Similarly, we observed disrupted Golgi morphology in the developing apical dendrite after maternal ethanol exposure which paralleled the morphological disruptions observed with 

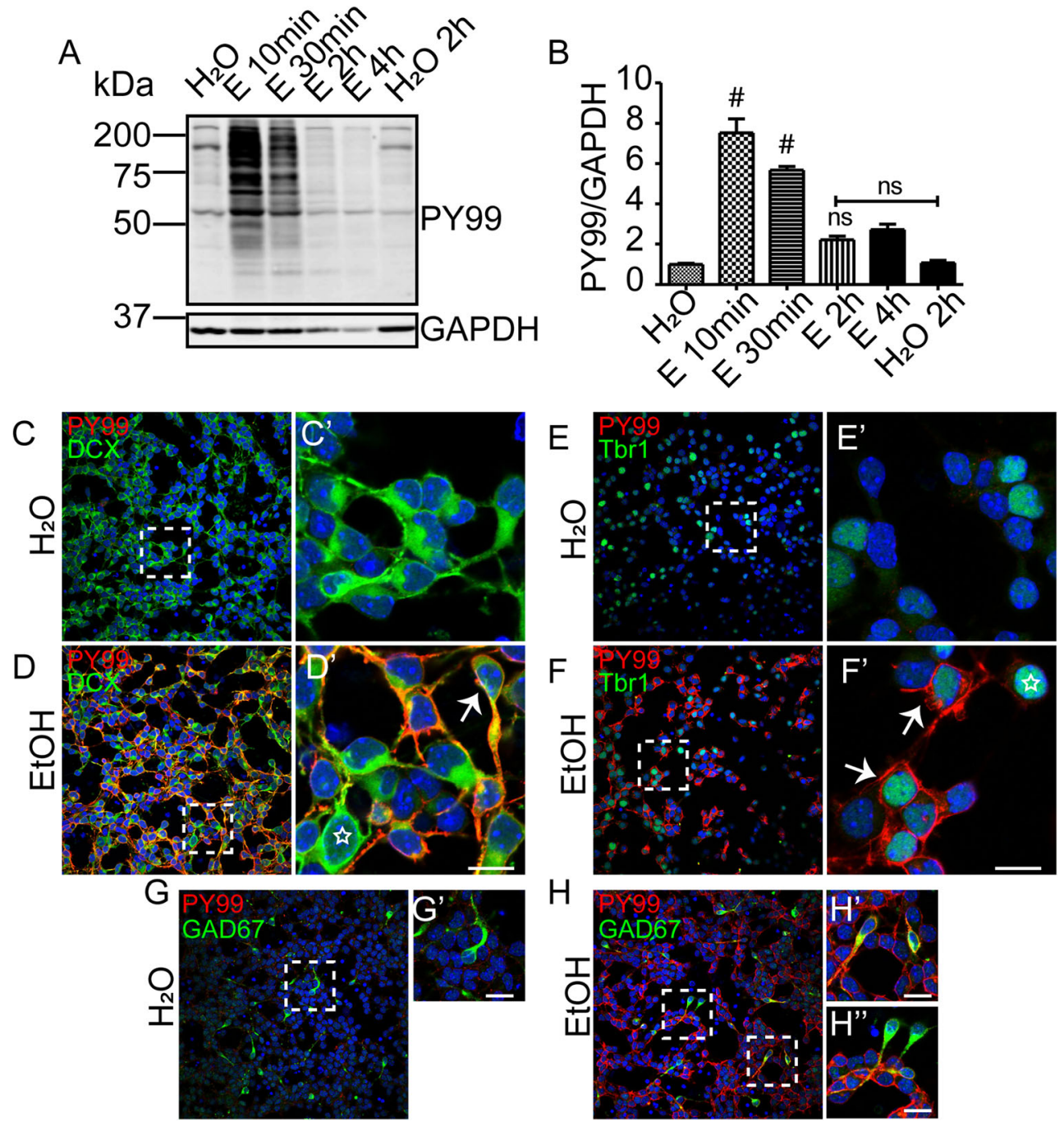

Fig. 5 Cultured cortical excitatory neurons and interneurons both respond to ethanol exposure. A Characterization of the time course and magnitude of the in vitro tyrosine phosphorylation response in E15 primary cortical culture after ethanol exposure. B Densitometric quantification of the response. $\mathbf{C}-\mathbf{F}$ Excitatory neurons respond to ethanol exposure. Primary cortical cultures were fixed and immunolabeled with anti-Doublecortin (Dcx) and pY99 after treatment with $\mathbf{C ~}_{2} \mathrm{O}$ or $\mathbf{D}$ ethanol ( $400 \mathrm{mg} / \mathrm{dL}$ ) for $10 \mathrm{~min}$. At higher magnification (dashed areas), little pY99 signal is observed in control (C'), but strong pY99 signal is observed in some (arrow) but not all (star) somata and neurites of $\mathrm{Dcx}^{+}$neurons (an average of $110 \mathrm{Dcx}^{+}$neurons were counted from 3 experiments) (D'). Similarly, E,F increased pY99 signal but not

Reelin-signaling disruptions [15, 42]. As Golgi and Golgi outposts perform critical functions in developing dendrite including microtubule stabilization, posttranslational protein modification, and transport as well as new membrane production $[35,36,51][37,52-54]$, this finding may provide insight into some of the sustained disruptions of dendritic function after fetal exposure [55, 56]. Although the SFK-dependent biochemical effects of the acute exposure appear to largely resolve by $4 \mathrm{~h}$ post treatment, neuronal damage may be more permanently encoded through sustained changes in Golgi

all $\mathrm{Tbr}^{+}$neurons (an average of $80 \mathrm{Tbr}^{+}$neurons were counted from 3 experiments) after ethanol exposure. Tbr1 is marker of excitatory cortical neurons at this time in development. F' The arrows indicate responding cells. The star indicates a non-responding cell. G-H GABAergic neurons respond to ethanol. pY99 immunostaining and GAD67 ${ }^{+}$immunostaining after 10 min of $\mathbf{G} \mathrm{H}_{2} \mathrm{O}$ or $\mathbf{H}$ ethanol exposure in E15 primary cortical culture reveal a subset of $\mathrm{GAD} 67^{+}$interneurons that are responsive to ethanol (an average of $\sim 160 \mathrm{GAD} 67^{+}$cells were counted from 3 experiments). Insets show higher magnification view of boxed regions in $(\mathbf{G})$ and $(\mathbf{H})$. Scale bar, $10 \mu \mathrm{m}$. Statistical determination by one-way ANOVA followed by Bonferroni's post hoc tests between groups. ${ }^{\#} p<0.001$

function and neurite outgrowth subsequent to SFK hyperactivation. Moreover, repeated acute exposures such as those that can be found with some forms of alcohol abuse disorder would be expected to cause successive periods of SFK disruption and, potentially, additive disruptions of neuronal development.

Disruption of SFK signaling may also impact neurite growth in other ways besides altering Reelin signaling [57] [45] [46] [47]. Src has important roles in both dendritic orientation and stabilization $[58,59]$ that may work in concert with 

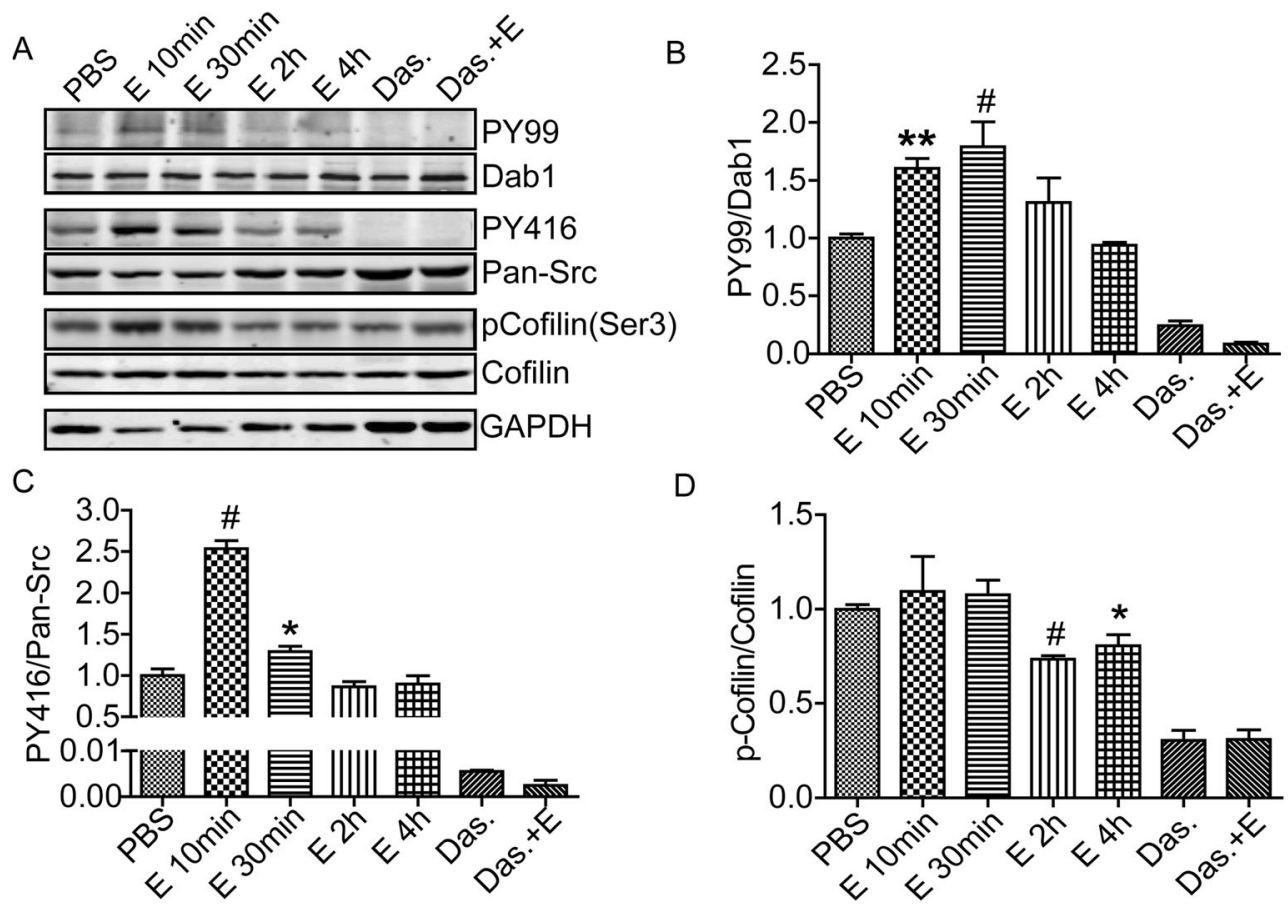

Fig. 6 Sustained disruption of Reelin signaling in fetal cortex by maternal ethanol exposure. A Western blot detection of tyrosine phosphorylation level of Reelin signaling adaptor protein Dab1, Src activation loop (pY416), and the actin severing protein n-cofilin in E15 embryonic cortical lysate cortex after PBS or 10, 30, 120, and $240 \mathrm{~min}$ of maternal ethanol exposure (i.p., $4 \mathrm{~g} / \mathrm{kg}$ ). B,C,D Corresponding densitometric quantification of blots reveals a sustained increase in tyrosine phosphorylation of Dab1 (B) as well as Src activation loop (pY416) (C). Both

Reelin-Dab1 signaling [60] [42] [40]. Neurite outgrowth and navigation is disrupted by ethanol [61] [62] [63] [64], and axonal growth can also depend on Src [47, 65, 66] [67]. Consistent with these findings, we observed that the intermediate zone and marginal zone, a major area of axonal and dendritic development respectively, also shows high Src expression and tyrosine phosphorylation in response to ethanol suggesting particular sensitivity to ethanol exposure.

The mechanism of ethanol-dependent Src activation is not clear: Ethanol activates a closely related SFK (Fyn) by inhibiting a regulatory STEP (striatal enriched phosphatase) $[68,69]$. However, this activation of Fyn occurred in striatal neurons and did not involve Src. In contrast, our prior study suggested that Fyn is not strongly activated in primary cortical neurons by ethanol [7]. Moreover, our prior study showed that preincubation with phenylarsine oxide (PAO), a membranepermeable inhibitor of class I phosphotyrosine phosphatases did not prevent ethanol-induced activation of Src in vitro. This suggests that phosphatase inhibition is unlikely to be the major mechanism of initial Src activation in the present study. Additional studies have found that ethanol may disrupt signaling in cholesterol-enriched lipid rafts [70] [71] including SFKcontaining rafts [72]. However, in our prior study, addition of cholesterol nor cholesterol depletion using methyl- $\beta$ -
$\mathrm{D}$

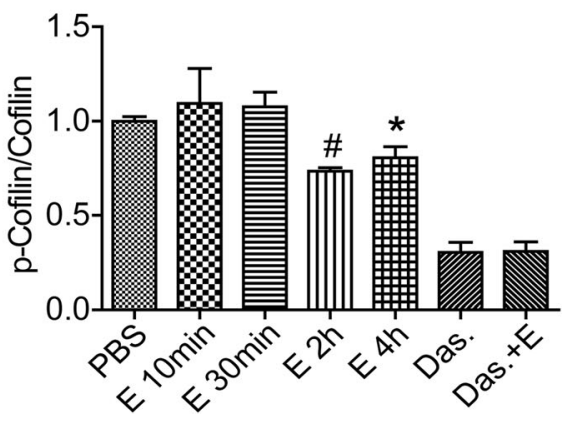

phosphorylation events are completely blocked by pretreatment of the dam with dasatinib $(20 \mathrm{mg} / \mathrm{kg}$ ). In addition, D Ser3 phosphorylation of the actin severing protein $\mathrm{n}$-cofilin starts to decline at $2 \mathrm{~h}$ after ethanol exposure. Densitometric values are first normalized the corresponding signal from total protein and then compared to PBS group. One-way ANOVA followed by Bonferroni's post hoc comparisons tests were used. ${ }^{*} p<0.05, * * p<0.01,{ }^{\#} p<0.001$

cyclodextrin did not dramatically alter the Src activation response of cultured neurons to ethanol [7]. Thus, the mechanism of Src activation in embryonic cortex by ethanol remains unclear.

Altered phosphatase activity functioning downstream of Src may be involved in the n-cofilin Ser3 dephosphorylation that is sustained for hours after initial ethanol exposure. While phosphatases are generally thought to be constitutively active and not highly regulated, regulatory mechanisms have been identified [73]. For example, as noted above, ethanol exposure can specifically inactivate STEP phosphatase [69]. There is also evidence that broad forms of cellular stress such as reactive oxygen species and ischemia can cause elevated tyrosine phosphatase activity [73] [74] raising the possibility of elevated phosphatase activity at the later time points after acute ethanol exposure. Slingshot homologs 1-3 (SSH 1-3) have been shown to dephosphorylate cofilin [75], and re-analysis of our prior transcriptional profiling study [76] as well as the Genepaint in situ database (https://gp3.mpg.de) shows that these phosphatases are expressed in the developing mouse cortex at E14.5, raising the possibility that their dysregulation may contribute to the later stage of the acute ethanol response. Alternatively, n-cofilin dephosphorylation may be due to the inhibition of kinases such as LIMK1 which 

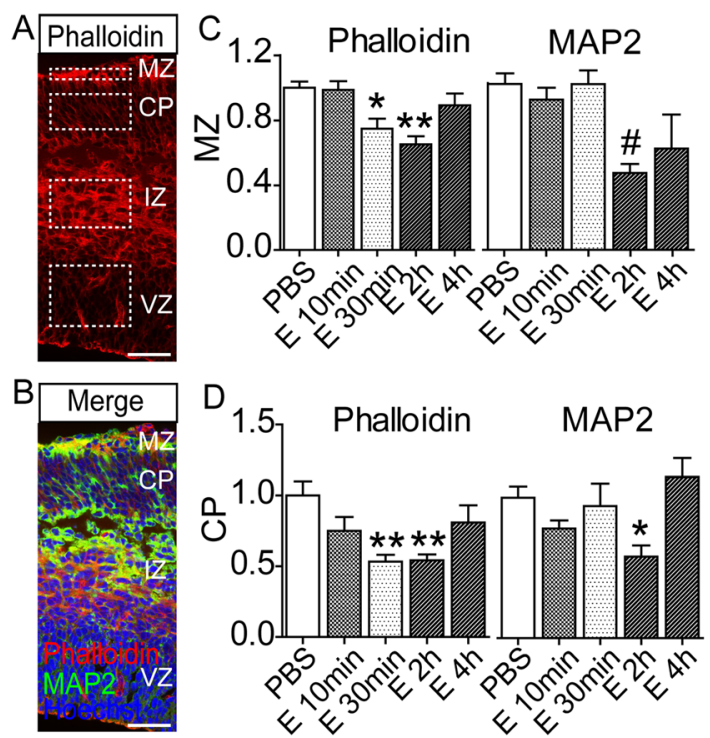

E 1.2 Phalloidin MAP2

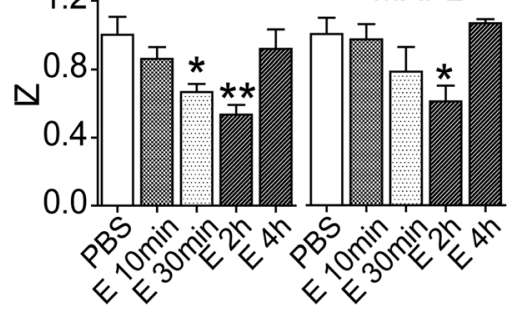

$\mathrm{F}$

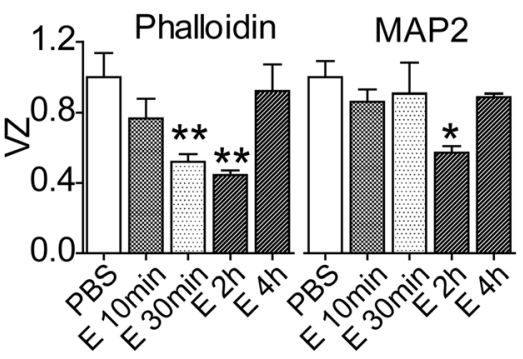

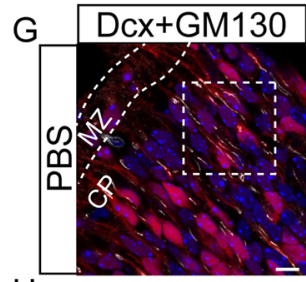
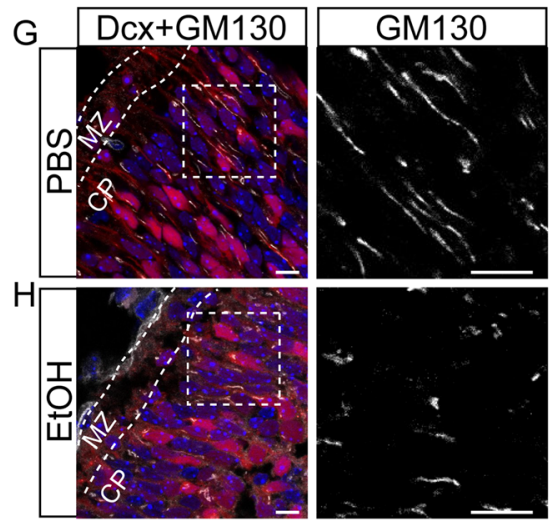

Fig. 7 Quantified mean immunofluorescence intensity of phalloidin and MAP2 signals, broken out by region and treatment. A-B Alexa555phalloidin and anti-MAP2 immunostaining in E15 cerebral cortex. Scale bar, $100 \mu \mathrm{m}$. C-F Significant reduction of the phalloidin signal intensity is observed across the cerebral wall by $30 \mathrm{~min}$ after ethanol exposure, whereas MAP2 signal intensity diminishes by $2 \mathrm{~h}$. Signal intensities were quantified from corresponding regions of interest identified in (A) (dashed rectangles). G-H GM130 immunostaining of E15 cortical sections derived from Dcx-dsRed ${ }^{14 \text { lu/J }}$ transgenic embryos after $4 \mathrm{~h}$ of

phosphorylate n-cofilin at Ser3 [77]. N-cofilin activity may be critical for both dendritic outgrowth [78] and to maintain Golgi morphology via a RhoA signaling pathway involving LIMK1, cofilin, and slingshot [79]. Understanding the balance of phosphatase and kinase activity during the persistent stage of the acute ethanol response will be critical to understanding the mechanisms of ethanol-induced signal suppression and may provide insight into the disrupted connectivity associated with fetal ethanol exposure.

Ethanol exposure causes broad disruptions to the developing nervous system [3-6], and explaining the diversity of ethanol's effects remains an important goal in fetal alcohol syndrome disorder (FASD) studies. This study raises the possibility that dysregulated Src activity may be an early response
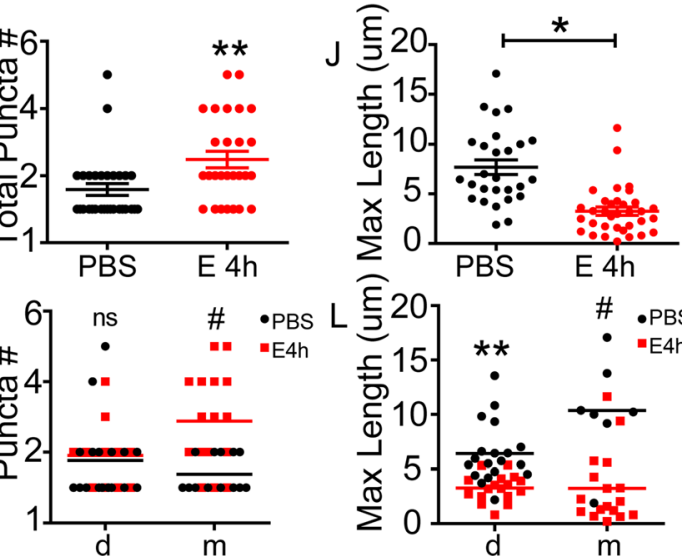

PBS or ethanol exposure. Scale bar, $10 \mu \mathrm{m}$. I-J Quantitation of total GM130 puncta number $(n=29$ cells for control and 27 cells for ethanol treated) and the maxim of the length ( $\mathrm{n}=27$ cells for control and 34 cells for ethanol) from cortical plate neurons. K-L Quantification of response broken by migratory and postmigratory neuronal morphology. Statistical determination by two-way ANOVA followed by Bonferroni's post hoc tests between groups. ${ }^{*} p<0.05, * * p<0.01,{ }^{*} p<0.001$. Abbreviations: $\mathrm{MZ}$, marginal zone; CP, cortical plate; IZ, intermediate zone; VZ, ventricular zone

to acute ethanol exposure and may be critical to understanding how ethanol disrupts multiple signaling pathways in the fetal brain. This work should enable further mapping of brain region and cell-type sensitivities to exposure as well as guide future studies of the genetic susceptibilities to ethanol-induced neural damage.

Supplementary Information The online version contains supplementary material available at https://doi.org/10.1007/s12035-021-02467-x.

Acknowledgements We thank Dr. Qiang Lu (Beckman Research Institute) for providing the Dcx-mRFP transgenic mice; we also thank Dr. Terrence Deak and Andrew Vore from Binghamton University for the assistance with the Analox BEC and AEC measurements. 
Author Contribution EO and DW designed the study with significant input from $\mathrm{BH}$. DW performed the study. EO and DW wrote the manuscript with comments and edits from $\mathrm{BH}$ and members of the Developmental Exposure to Alcohol Research Center (DEARC).

Funding This work is supported by the National Institute on Alcohol Abuse and Alcoholism (NIAAA) through Main Project \#1 of the Developmental Exposure to Alcohol Research Center (DEARC) P50AA017823-06. Continuing support is provided by the Hendrick's Fund (SUNY Upstate Medical University).

Data Availability All authors agree that all data and materials as well as software application comply with field standards.

Code Availability Not applicable.

\section{Declarations}

Ethics Approval Not applicable.

Consent to Participate Not applicable.

Consent for Publication The authors guarantees that the contribution to the work has not been previously published elsewhere and the authors declare that any person named as co-author of the contribution is aware of the fact and has agreed to being so named.

Conflict of Interest The authors declare no competing interests.

Open Access This article is licensed under a Creative Commons Attribution 4.0 International License, which permits use, sharing, adaptation, distribution and reproduction in any medium or format, as long as you give appropriate credit to the original author(s) and the source, provide a link to the Creative Commons licence, and indicate if changes were made. The images or other third party material in this article are included in the article's Creative Commons licence, unless indicated otherwise in a credit line to the material. If material is not included in the article's Creative Commons licence and your intended use is not permitted by statutory regulation or exceeds the permitted use, you will need to obtain permission directly from the copyright holder. To view a copy of this licence, visit http://creativecommons.org/licenses/by/4.0/.

\section{References}

1. May PA, Chambers CD, Kalberg WO, Zellner J, Feldman H, Buckley D, Kopald D, Hasken JM et al (2018) Prevalence of fetal alcohol spectrum disorders in 4 US communities. JAMA 319:474482

2. Streissguth AP, Barr HM, Kogan J, Bookstein FL (1996) Alcohol syndrome (FAS) and fetal alcohol effects (FAE)," Final report to the centers for disease control and prevention (CDC)". University of Washington, Fetal Alcohol \& Drug Unit, Tech Rep No 96-06.

3. Donald KA, Eastman E, Howells FM, Adnams C, Riley EP, Woods RP, Narr KL, Stein DJ (2015) Neuroimaging effects of prenatal alcohol exposure on the developing human brain: a magnetic resonance imaging review. Acta Neuropsychiatr 27:251-269

4. Jarmasz JS, Basalah DA, Chudley AE, Del Bigio MR (2017) Human brain abnormalities associated with prenatal alcohol exposure and fetal alcohol spectrum disorder. J Neuropathol Exp Neurol $76: 813-833$
5. Riley EP, Mattson SN, Sowell ER, Jernigan TL, Sobel DF, Jones KL (1995) Abnormalities of the corpus callosum in children prenatally exposed to alcohol. Alcohol Clin Exp Res 19:1198-1202

6. Yang Y, Phillips OR, Kan E, Sulik KK, Mattson SN, Riley EP, Jones KL, Adnams CM et al (2012) Callosal thickness reductions relate to facial dysmorphology in fetal alcohol spectrum disorders. Alcohol Clin Exp Res 36:798-806

7. Wang D, Enck J, Howell BW, Olson EC (2019) Ethanol exposure transiently elevates but persistently inhibits tyrosine kinase activity and impairs the growth of the nascent apical dendrite. Mol Neurobiol 56:5749-5762

8. Caviness VS Jr, Sidman RL (1973) Time of origin or corresponding cell classes in the cerebral cortex of normal and reeler mutant mice: an autoradiographic analysis. J Comp Neurol 148:141-151

9. Hong SE, Shugart YY, Huang DT, Shahwan SA, Grant PE, Hourihane JO, Martin ND, Walsh CA (2000) Autosomal recessive lissencephaly with cerebellar hypoplasia is associated with human RELN mutations. Nat Genet 26:93-96

10. D ' Arcangelo G, Miao GG, Chen SC, Soares HD, Morgan JI, Curran T (1995) A protein related to extracellular matrix proteins deleted in the mouse mutant reeler. Nature 374:719-723

11. Hirotsune S, Takahara T, Sasaki N, Hirose K, Yoshiki A, Ohashi T, Kusakabe M, Murakami Y et al (1995) The reeler gene encodes a protein with an EGF-like motif expressed by pioneer neurons. Nat Genet 10:77-83

12. Trommsdorff M, Gotthardt M, Hiesberger T, Shelton J, Stockinger W, Nimpf J, Hammer RE, Richardson JA et al (1999) Reeler/ Disabled-like disruption of neuronal migration in knockout mice lacking the VLDL receptor and ApoE receptor 2. Cell 97:689-701

13. Howell BW, Hawkes R, Soriano P, Cooper JA (1997) Neuronal position in the developing brain is regulated by mouse disabled-1. Nature 389:733-737

14. Kuo G, Arnaud L, Kronstad-O ' Brien P, Cooper JA (2005) Absence of Fyn and Src causes a reeler-like phenotype. J Neurosci 25:8578-8586

15. O ' Dell RS, Ustine CJ, Cameron DA, Lawless SM, Williams RR, Zipfel WR, Olson EC (2012) Layer 6 cortical neurons require Reelin-Dab1 signaling for cellular orientation, Golgi deployment, and directed neurite growth into the marginal zone. Neural development 7:25

16. Powrozek TA, Olson EC (2012) Ethanol-induced disruption of Golgi apparatus morphology, primary neurite number and cellular orientation in developing cortical neurons. Alcohol 46:619-627

17. Gleeson JG, Lin PT, Flanagan LA, Walsh CA (1999) Doublecortin is a microtubule-associated protein and is expressed widely by migrating neurons. Neuron 23:257-271

18. Wang X, Qiu R, Tsark W, Lu Q (2007) Rapid promoter analysis in developing mouse brain and genetic labeling of young neurons by doublecortin-DsRed-express. J Neurosci Res 85:3567-3573

19. D ' Souza El-Guindy NB, Kovacs EJ, De Witte P, Spies C, Littleton JM, de Villiers WJ, Lott AJ, Plackett TP et al (2010) Laboratory models available to study alcohol-induced organ damage and immune variations: choosing the appropriate model. Alcohol Clin Exp Res 34:1489-1511

20. Majchrowicz E (1975) Induction of physical dependence upon ethanol and the associated behavioral changes in rats. Psychopharmacologia 43:245-254

21. Wong EL, Lutz NM, Hogan VA, Lamantia CE, McMurray HR, Myers JR, Ashton JM, Majewska AK (2018) Developmental alcohol exposure impairs synaptic plasticity without overtly altering microglial function in mouse visual cortex. Brain Behav Immun 67:257-278

22. Afshar M, Netzer G, Salisbury-Afshar E, Murthi S, Smith GS (2016) Injured patients with very high blood alcohol concentrations. Injury 47:83-88 
23. Hamlyn AN, Brown AJ, Sherlock S, Baron DN (1975) Casual blood-ethanol estimations in patients with chronic liver disease. Lancet 2:345-347

24. Belknap JK, Crabbe JC, Young ER (1993) Voluntary consumption of ethanol in 15 inbred mouse strains. Psychopharmacology (Berl) 112:503-510

25. Goodlett CR, Gilliam DM, Nichols JM, West JR (1989) Genetic influences on brain growth restriction induced by development exposure to alcohol. Neurotoxicology 10:321-334

26. Olney JW, Tenkova T, Dikranian K, Muglia LJ, Jermakowicz WJ, D ' Sa C, Roth KA (2002) Ethanol-induced caspase-3 activation in the in vivo developing mouse brain. Neurobiol Dis 9:205-219

27. Lombardo LJ, Lee FY, Chen P, Norris D, Barrish JC, Behnia K, Castaneda S, Cornelius LAM et al (2004) Discovery of N-(2chloro-6-methyl- phenyl)-2-(6-(4-(2-hydroxyethyl)- piperazin-1yl)-2-methylpyrimidin-4- ylamino)thiazole-5-carboxamide (BMS354825), a dual Src/Abl kinase inhibitor with potent antitumor activity in preclinical assays. J Med Chem 47:6658-6661

28. Shah NP, Tran C, Lee FY, Chen P, Norris D, Sawyers CL (2004) Overriding imatinib resistance with a novel ABL kinase inhibitor. Science 305:399-401

29. Steinberg M (2007) Dasatinib: a tyrosine kinase inhibitor for the treatment of chronic myelogenous leukemia and Philadelphia chromosome-positive acute lymphoblastic leukemia. Clin Ther 29:2289-2308

30. Nichols AJ, O ' Dell RS, Powrozek TA, Olson EC (2013) Ex utero electroporation and whole hemisphere explants: a simple experimental method for studies of early cortical development. Journal of visualized experiments, JoVE

31. Englund C, Fink A, Lau C, Pham D, Daza RA, Bulfone A, Kowalczyk T, Hevner RF (2005) Pax6, Tbr2, and Tbr1 are expressed sequentially by radial glia, intermediate progenitor cells, and postmitotic neurons in developing neocortex. J Neurosci 25: 247-251

32. Chai X, Forster E, Zhao S, Bock HH, Frotscher M (2009b) Reelin acts as a stop signal for radially migrating neurons by inducing phosphorylation of $\mathrm{n}$-cofilin at the leading edge. Commun Integr Biol 2:375-377

33. Kim YB, Choi S, Choi MC, Oh MA, Lee SA, Cho M, Mizuno K, Kim SH et al (2008) Cell adhesion-dependent cofilin serine 3 phosphorylation by the integrin-linked kinase.c-Src complex. J Biol Chem 283:10089-10096

34. Moriyama K, Iida K, Yahara I (1996) Phosphorylation of Ser-3 of cofilin regulates its essential function on actin. Genes Cells 1:73-86

35. Horton AC, Ehlers MD (2003) Dual modes of endoplasmic reticulum-to-Golgi transport in dendrites revealed by live-cell imaging. J Neurosci 23:6188-6199

36. Horton AC, Racz B, Monson EE, Lin AL, Weinberg RJ, Ehlers MD (2005) Polarized secretory trafficking directs cargo for asymmetric dendrite growth and morphogenesis. Neuron 48:757-771

37. Yang SZ, Wildonger J (2020) Golgi outposts locally regulate microtubule orientation in neurons but are not required for the overall polarity of the dendritic cytoskeleton. Genetics. 215:435-447

38. Weller SG, Capitani M, Cao H, Micaroni M, Luini A, Sallese M, McNiven MA (2010) Src kinase regulates the integrity and function of the Golgi apparatus via activation of dynamin 2. Proc Natl Acad Sci U S A 107:5863-5868

39. Romero AM, Renau-Piqueras J, Marin MP, Esteban-Pretel G (2015) Chronic alcohol exposure affects the cell components involved in membrane traffic in neuronal dendrites. Neurotox Res 27 : 43-54

40. Matsuki T, Matthews RT, Cooper JA, van der Brug MP, Cookson MR, Hardy JA, Olson EC, Howell BW (2010) Reelin and stk25 have opposing roles in neuronal polarization and dendritic Golgi deployment. Cell 143:826-836
41. Meseke M, Rosenberger G, Forster E (2013) Reelin and the Cdc42/ Rac1 guanine nucleotide exchange factor alphaPIX/Arhgef6 promote dendritic Golgi translocation in hippocampal neurons. Eur J Neurosci 37:1404-1412

42. Nichols AJ, Olson EC (2010) Reelin promotes neuronal orientation and dendritogenesis during preplate splitting. Cerebral cortex 20: 2213-2223

43. Hiesberger T, Trommsdorff M, Howell BW, Goffinet A, Mumby MC, Cooper JA, Herz J (1999) Direct binding of Reelin to VLDL receptor and ApoE receptor 2 induces tyrosine phosphorylation of disabled-1 and modulates tau phosphorylation. Neuron 24:481-489

44. Howell BW, Herrick TM, Cooper JA (1999) Reelin-induced tryosine phosphorylation of disabled 1 during neuronal positioning. Genes Dev 13:643-648

45. Murphy DA, Diaz B, Bromann PA, Tsai JH, Kawakami Y, Maurer J, Stewart RA, Izpisua-Belmonte JC et al (2011) A Src-Tks5 pathway is required for neural crest cell migration during embryonic development. PLoS One 6:e22499

46. Knoll B, Drescher U (2004) Src family kinases are involved in EphA receptor-mediated retinal axon guidance. J Neurosci 24: $6248-6257$

47. Liu G, Beggs H, Jurgensen C, Park HT, Tang H, Gorski J, Jones KR, Reichardt LF et al (2004) Netrin requires focal adhesion kinase and Src family kinases for axon outgrowth and attraction. Nature neuroscience 7:1222-1232

48. Soriano P, Montgomery C, Geske R, Bradley A (1991) Targeted disruption of the c-src proto-oncogene leads to osteopetrosis in mice. Cell 64:693-702

49. Jossin Y, Ogawa M, Metin C, Tissir F, Goffinet AM (2003) Inhibition of SRC family kinases and non-classical protein kinases $\mathrm{C}$ induce a reeler-like malformation of cortical plate development. J Neurosci 23:9953-9959

50. Chai X, Forster E, Zhao S, Bock HH, Frotscher M (2009a) Reelin stabilizes the actin cytoskeleton of neuronal processes by inducing n-cofilin phosphorylation at serine3. J Neurosci 29:288-299

51. Ehlers MD (2007) Secrets of the secretory pathway in dendrite growth. Neuron 55:686-689

52. Lewis TL Jr, Polleux F (2012) Neuronal morphogenesis: Golgi outposts, acentrosomal microtubule nucleation, and dendritic branching. Neuron 76:862-864

53. Ori-McKenney KM, Jan LY, Jan YN (2012) Golgi outposts shape dendrite morphology by functioning as sites of acentrosomal microtubule nucleation in neurons. Neuron 76:921-930

54. Winkle CC, Gupton SL (2016) Membrane trafficking in neuronal development: ins and outs of neural connectivity. Int Rev Cell Mol Biol 322:247-280

55. Lawrence RC, Otero NK, Kelly SJ (2012) Selective effects of perinatal ethanol exposure in medial prefrontal cortex and nucleus accumbens. Neurotoxicology and teratology 34:128-135

56. Louth EL, Luctkar HD, Heney KA, Bailey CDC (2018) Developmental ethanol exposure alters the morphology of mouse prefrontal neurons in a layer-specific manner. Brain Res 1678:94 105

57. Parsons SJ, Parsons JT (2004) Src family kinases, key regulators of signal transduction. Oncogene 23:7906-7909

58. Buhusi M, Midkiff BR, Gates AM, Richter M, Schachner M, Maness PF (2003) Close homolog of L1 is an enhancer of integrin-mediated cell migration. The Journal of biological chemistry 278:25024-25031

59. Demyanenko GP, Schachner M, Anton E, Schmid R, Feng G, Sanes J, Maness PF (2004) Close homolog of L1 modulates areaspecific neuronal positioning and dendrite orientation in the cerebral cortex. Neuron 44:423-437

60. Niu S, Renfro A, Quattrocchi CC, Sheldon M, D ' Arcangelo G (2004) Reelin promotes hippocampal dendrite development through the VLDLR/ApoER2-Dab1 pathway. Neuron 41:71-84 
61. Bearer CF, Swick AR, O ' Riordan MA, Cheng G (1999) Ethanol inhibits L1-mediated neurite outgrowth in postnatal rat cerebellar granule cells. J Biol Chem 274:13264-13270

62. Lindsley TA, Kerlin AM, Rising LJ (2003) Time-lapse analysis of ethanol 's effects on axon growth in vitro. Brain research Developmental brain research 147:191-199

63. Lindsley TA, Miller MW, Littner Y, Bearer CF (2006) Signaling pathways regulating cell motility: a role in ethanol teratogenicity? Alcohol Clin Exp Res 30:1445-1450

64. Yanni PA, Lindsley TA (2000) Ethanol inhibits development of dendrites and synapses in rat hippocampal pyramidal neuron cultures. Brain research Developmental brain research 120:233-243

65. Atashi JR, Klinz SG, Ingraham CA, Matten WT, Schachner M, Maness PF (1992) Neural cell adhesion molecules modulate tyrosine phosphorylation of tubulin in nerve growth cone membranes. Neuron 8:831-842

66. Maness PF, Schachner M (2007) Neural recognition molecules of the immunoglobulin superfamily: signaling transducers of axon guidance and neuronal migration. Nature neuroscience 10:19-26

67. Schmid RS, Pruitt WM, Maness PF (2000) A MAP kinasesignaling pathway mediates neurite outgrowth on L1 and requires Src-dependent endocytosis. The Journal of neuroscience : the official journal of the Society for Neuroscience 20:4177-4188

68. Nguyen TH, Liu J, Lombroso PJ (2002) Striatal enriched phosphatase 61 dephosphorylates Fyn at phosphotyrosine 420. J Biol Chem 277:24274-24279

69. Xu J, Kurup P, Foscue E, Lombroso PJ (2015) Striatal-enriched protein tyrosine phosphatase regulates the PTPalpha/Fyn signaling pathway. J Neurochem 134:629-641

70. Szabo G, Dolganiuc A, Dai Q, Pruett SB (2007) TLR4, ethanol, and lipid rafts: a new mechanism of ethanol action with implications for other receptor-mediated effects. J Immunol 178:1243-1249

71. Huang SS, Chen CL, Huang FW, Johnson FE, Huang JS (2016) Ethanol enhances TGF-beta activity by recruiting TGF-beta receptors from intracellular vesicles/lipid rafts/caveolae to nonlipid raft microdomains. J Cell Biochem 117:860-871

72. Vacaresse N, Moller B, Danielsen EM, Okada M, Sap J (2008) Activation of c-Src and Fyn kinases by protein-tyrosine phosphatase RPTPalpha is substrate-specific and compatible with lipid raft localization. J Biol Chem 283:35815-35824

73. Gingras AC (2011) Protein phosphatases, from molecules to networks. EMBO Rep 12:1211-1213

74. Pan KT, Chen YY, Pu TH, Chao YS, Yang CY, Bomgarden RD, Rogers JC, Meng TC et al (2014) Mass spectrometry-based quantitative proteomics for dissecting multiplexed redox cysteine modifications in nitric oxide-protected cardiomyocyte under hypoxia. Antioxid Redox Signal 20:1365-1381

75. Ohashi K (2015) Roles of cofilin in development and its mechanisms of regulation. Dev Growth Differ 57:275-290

76. Cameron DA, Middleton FA, Chenn A, Olson EC (2012) Hierarchical clustering of gene expression patterns in the Eomes + lineage of excitatory neurons during early neocortical development. BMC Neurosci 13:90

77. Arber S, Barbayannis FA, Hanser H, Schneider C, Stanyon CA, Bernard O, Caroni P (1998) Regulation of actin dynamics through phosphorylation of cofilin by LIM-kinase. Nature 393:805-809

78. Lauterborn JC, Kramar EA, Rice JD, Babayan AH, Cox CD, Karsten CA, Gall CM, Lynch G (2017) Cofilin activation is temporally associated with the cessation of growth in the developing hippocampus. Cereb Cortex 27:2640-2651

79. Quassollo G, Wojnacki J, Salas DA, Gastaldi L, Marzolo MP, Conde C, Bisbal M, Couve A et al (2015) A RhoA signaling pathway regulates dendritic Golgi outpost formation. Curr Biol 25:971982

Publisher's Note Springer Nature remains neutral with regard to jurisdictional claims in published maps and institutional affiliations. 